\title{
Induced PUA representations and selection rules
}

\author{
P M van den Broek \\ Institute for Theoretical Physics, University of Nijmegen, Toernooiveld, Nijmegen, the \\ Netherlands
}

Received 29 September 1977, in final form 19 December 1977

\begin{abstract}
Well known properties of induced unitary representations are generalised for induced PUA representations. They are applied to obtain an expression for the multiplicities in the Clebsch-Gordon series of induced pUA representations. Finally we show how these multiplicities can be used for the determination of selection rules.
\end{abstract}

\section{Introduction}

In the quantum mechanical description of a physical system with symmetry group $G$ the Hilbert space $\mathscr{H}$ of state vectors carries a projective unitary-anti-unitary (PUA) representation $T$ of $G$, according to the well known results of Wigner (1939) and Bargmann (1954). As a basis for $\mathscr{H}$ we may choose vectors $\phi_{m}^{\rho, a}$, transforming under $G$ as basis vectors of irreducible PUA representations $D^{\circ}$ of $G$ :

$$
T(g) \phi_{m}^{\rho, a}=\sum_{n} D_{n m}^{\rho}(g) \phi_{n}^{\rho, a} \quad \forall g \in G .
$$

Here $\rho$ labels the irreducible PUA representations of $G$ which have the same factor system as $T$, the index $a$ denotes that there may be more than one set of basis functions transforming according to $D^{\rho}$, and $m$ and $n$ have values from one up to the dimension of $D^{\rho}$. All relevant physical information, such as transition probabilities, is contained in matrix elements $\left(\phi_{m}^{\rho, a}, O_{k}^{\mu} \phi_{l}^{\nu, b}\right)$, where $O^{\mu}$ is an irreducible tensor operator transforming according to the irreducible UA representation $D^{\mu}$ of $G$ :

$$
T(g) O_{k}^{\mu} T(g)^{-1}=\sum_{n} D_{n k}^{\mu}(g) O_{n}^{\mu} \quad \forall g \in G .
$$

In order to study the properties, due to the symmetry group $G$, of these matrix elements one has to study the PUA representations of $G$. Analogous to the well known selection rules for unitary representations the matrix elements $\left(\phi_{m}^{o, a}, O_{k}^{\mu} \phi_{l}^{\nu, b}\right)$ are zero for each $m, k, l, a$ and $b$ if the trivial UA representation of $G$ does not occur in the triple direct product $D^{\rho^{*}} \otimes D^{\mu} \otimes D^{\nu}$. This will be shown in $\S 5$.

An important example of a physical system where one has to use projective unitary representations instead of ordinary unitary representations is an electron in a crystalline solid with a uniform magnetic field (Brown 1964, Tam 1969). Moreover, whenever an element $g$ of $G$ contains the time reversal operation, $T(g)$ must be an anti-unitary operator. This shows that the study of PUA representations is of general importance for physics. 
It often occurs, for instance if $G$ is a Shubnikov space group, that one has to obtain the PUA representations of $G$ with the help of an induction procedure. The induction procedure for unitary representations, which goes back to Frobenius, is by now well known. (See for instance Coleman (1966) and Bradley (1966).) This induction procedure has recently been generalised for PUA representations by Shaw and Lever (1974). The aim of this paper is to study general properties of induced PUA representations and to apply these properties to the study of selection rules. In $\S 3$ we generalise some well known properties of induced unitary representations, such as for instance the Frobenius reciprocity theorem and Mackey's subgroup theorem. In $\S 4$ we use these results to obtain an expression for the multiplicities in the ClebschGordan series of induced PUA representations. Finally in $\$ 5$ we discuss selection rules, using the results of the preceding sections.

\section{Preliminaries}

Let $G$ be a finite group and $G_{0}$ a subgroup of index 1 or 2 . A PUA representation of $G$ with respect to $G_{0}$ is a mapping $D$ from $G$ into the operators on some Hilbert space $\mathscr{H}$ such that:

(i) $D(g)$ is unitary if $g \in G_{0}$ and anti-unitary if $g \notin G_{0}$.

(ii) $D(g) D\left(g^{\prime}\right)=\sigma\left(g, g^{\prime}\right) D\left(g g^{\prime}\right)$ for all $g, g^{\prime} \in G$ for some mapping $\sigma: G \times G \rightarrow$ $U(1)$.

(iii) $D(e)=I$, where $e$ is the identity of $G$ and $I$ is the identity operator on $\mathscr{H}$.

The mapping $\sigma$ is called a factor system of $G$ with respect to $G_{0}$ and satisfies

and

$$
\sigma(g, e)=\sigma(e, g)=1 \quad \forall g \in G
$$

$$
\sigma\left(g, g^{\prime}\right) \sigma\left(g g^{\prime}, g^{\prime \prime}\right)=\sigma\left(g, g^{\prime} g^{\prime \prime}\right) \sigma^{g}\left(g^{\prime}, g^{\prime \prime}\right) \quad \forall g, g^{\prime}, g^{\prime \prime} \in G
$$

where $\lambda^{\mathbf{g}}$ is defined by

$$
\lambda^{g}= \begin{cases}\lambda & \text { if } g \in G_{0} \\ \lambda^{*} & \text { if } g \notin G_{0}\end{cases}
$$

and the asterisk denotes complex conjugation.

In the following we shall drop the phrase 'with respect to ...' wherever it is clear which the appropriate subgroup of index 1 or 2 is. Suppose $\mathscr{H}$ is finite-dimensional and let $\left\{e_{1}, \ldots, e_{n}\right\}$ be an orthonormal basis of $\mathscr{H}$. For each operator $D(g)$ a unitary $n \times n$ matrix $\mathscr{D}(g)$ is defined by $\mathscr{D}_{i j}(g)=\left(e_{i}, D(g) e_{j}\right)$. $G$ :

The matrices $\mathscr{D}(g)$ form a PUA matrix representation or PUAM representation of

$$
\mathscr{D}(g) \mathscr{D}^{g}\left(g^{\prime}\right)=\sigma\left(g, g^{\prime}\right) \mathscr{D}\left(g g^{\prime}\right)
$$

where $\mathscr{D}^{8}$ is defined by

$$
\mathscr{D}^{\mathbb{8}}= \begin{cases}\mathscr{D} & \text { if } g \in G_{0} \\ \mathscr{D}^{*} & \text { if } g \notin G_{0} .\end{cases}
$$

A PUA representation $D$ of $G$ is reducible if there is a proper subspace $\mathscr{H}^{\prime}$ of $\mathscr{H}$ which is invariant under $D(G)$; otherwise $D$ is irreducible. The direct product $D_{1} \times D_{2}$ of PUA representations $D_{1}$ and $D_{2}$ of $G$ with factor systems $\sigma_{1}$ and $\sigma_{2}$ respectively is a 
PUA representation of $G$ with factor system $\sigma_{1} \sigma_{2}$ and is defined by

$$
\left(\mathscr{D}_{1} \times \mathscr{D}_{2}\right)(g)_{i j, k l}=\mathscr{D}_{1}(g)_{i k} \mathscr{D}_{2}(g)_{j l} \text {. }
$$

Two PUA representations $D_{1}$ and $D_{2}$ of $G$ are equivalent if there exists a unitary matrix $S$ such that

$$
\mathscr{D}_{1}(g)=S^{-1} \mathscr{D}_{2}(g) S^{g} \quad \forall g \in G
$$

or, equivalently, if

$$
\operatorname{Tr}\left\{\mathscr{D}_{1}(g)\right\}=\operatorname{Tr}\left\{\mathscr{D}_{2}(g)\right\} \quad \forall g \in G_{0} .
$$

In the following we shall identify equivalent PUA representations, and also identify a PUA representation with the corresponding PUAM representation. Suppose $H$ is a subgroup of $G$, and let $g_{1}=e, g_{2}, \ldots, g_{p}$ be left coset representatives of $G$ with respect to $H$. Then $G=\sum_{i=1}^{p} g_{i} H$. Let $\sigma$ be a factor system of $G$ and let $\Delta$ be a PUA representation of $H$ with respect to $H_{0}$ with factor system $\sigma$, where $H_{0}=H \cap G_{0}$. Then $\Delta_{\sigma}^{\dagger} G$ is the pUA representation of $G$ with factor system $\sigma$ which is induced from $\Delta$ and is defined by

$$
\left(\Delta_{\sigma}^{\uparrow} G\right)(g)_{j t, k s}= \begin{cases}0 & \text { if } g_{j}^{-1} g g_{k} \notin H \\ \sigma\left(g, g_{k}\right) \sigma^{*}\left(g_{j}, g_{j}^{-1} g g_{k}\right) \Delta^{g_{j}}\left(g_{j}^{-1} g g_{k}\right)_{t s} & \text { if } g_{j}^{-1} g g_{k} \in H .\end{cases}
$$

It is straightforward calculation to verify that $\Delta_{\sigma}^{\uparrow} G$ does not depend on the choice of the left coset representatives. By convention, $\Delta \uparrow G$ will be an induced UA representation.

\section{Properties of induced PUA representations}

The number of times an irreducible PUA representation $D^{\mu}$ occurs in a PUA representation $D$, which we will denote by $i\left(D^{\mu}, D\right)$, is given by

$$
i\left(D^{\mu}, D\right)=\frac{\Sigma_{g \in G_{0}} \chi^{\mu}(g) \chi^{*}(g)}{\sum_{g \in G_{0}} \chi^{\mu}(g) \chi^{\mu}(g)^{*}}
$$

where $\chi^{\mu}$ and $\chi$ are the characters of $D^{\mu}$ and $D$ respectively. This property has been given by Karavaev (1965) for UA representations and can easily be shown to hold for PUA representations as well, independent of the factor system involved. An immediate consequence is

$$
i\left(D^{\mu}, D\right)=\frac{i\left(D^{0}, D \otimes D^{\mu^{*}}\right)}{\left|1 / G_{0}\right| \Sigma_{g \in G_{0}} \chi^{\mu}(g) \chi^{\mu^{*}}(g)}
$$

where $D^{0}$ is the trivial UA representation of $G$ and $\left|\mathrm{G}_{0}\right|$ denotes the order of $G_{0}$. In the remainder of this section $\omega$ and $\sigma$ will be factor systems of the finite group $G, H$ will be a subgroup of $G$, and $D^{0}(H)$ and $D^{\circ}(G)$ will be the trivial UA representations of $H$ and $G$ respectively.

Theorem 1. Let $D$ be a UA representation of $H$. Then

$$
i\left(D^{\circ}(H), D\right)=\frac{|H|}{\left|H_{0}\right|} \frac{\left|G_{0}\right|}{|G|} i\left(D^{\circ}(G), D \uparrow G\right) .
$$


Proof. Define $\delta(g)$ by $\delta(g)=1$ if $g \in H$ and $\delta(g)=0$ if $g \notin H$; let $\psi$ and $\chi$ be the characters of $D$ and $D \uparrow G$ respectively. Then

$$
\begin{aligned}
& i\left(D^{\circ}(G), D \uparrow G\right)=\frac{1}{\left|G_{0}\right|} \sum_{\mathbf{g} \in G_{0}} \chi(g)=\frac{1}{\left|G_{0}\right|} \sum_{g \in G_{0}} \sum_{i j} D_{i j}^{g_{i}}\left(g_{i}^{-1} g g_{i}\right) \delta\left(g_{i}^{-1} g g_{i}\right) \\
& =\frac{1}{\left|G_{0}\right|} \sum_{i} \sum_{g \in G_{0}} \psi^{g_{i}}\left(g_{i}^{-1} g g_{i}\right) \delta\left(g_{i}^{-1} g g_{i}\right)=\frac{1}{\left|G_{0}\right|} \sum_{i} \sum_{g \in H_{0}} \psi^{g_{i}}(g) \\
& =\frac{\left|H_{0}\right|}{\left|G_{0}\right|} \frac{|G|}{|H|} i\left(D^{0}(H), D\right) \text {. }
\end{aligned}
$$

Theorem 2. Let $\Delta$ be a PUA representation of $H$ with factor system $\sigma$ and $\Gamma$ a PUA representation of $G$ with factor system $\omega$. Then

$$
\Gamma \otimes(\Delta \underset{\sigma}{\uparrow} G)=[(\Gamma \downarrow H) \otimes \Delta] \uparrow_{\omega \sigma} G .
$$

Proof. We have to show that $\operatorname{Tr}\left\{\left[\Gamma \otimes\left(\Delta_{\sigma}^{\uparrow} G\right)\right](g)\right\}$ and $\operatorname{Tr}\left\{\left[((\Gamma \downarrow H) \otimes \Delta)_{\omega \sigma}^{\uparrow} G\right](g)\right\}$ are equal for each $g \in G_{0}$. Evaluating both terms we see that this' is true if $\chi(g) \omega^{*}\left(g, g_{j}\right) \omega\left(g_{j}, g_{i}^{-1} g g_{j}\right)=\chi^{g_{i}}\left(g_{j}^{-1} g g_{j}\right)$ for each $j$ and each $g \in G_{0}$ for which $g_{i}^{-1} g g_{j}$ belongs to $H$, if $\chi$ is the character of $\Gamma$. But this follows from

$$
\Gamma\left(g_{j}\right) \Gamma^{\mathbf{g}_{i}}\left(g_{i}^{-1} g g_{j}\right) \Gamma^{g_{j}}\left(g_{j}^{-1}\right)=\omega\left(g_{j}, g_{j}^{-1} g g_{j}\right) \omega\left(g g_{j}, g_{j}^{-1}\right) \Gamma(g)
$$

if we take the trace of both sides and use the equations (2.1) and (2.2).

Theorem 3 (Frobenius reciprocity theorem). Let $\Gamma$ and $\Delta$ be irreducible PUA representations of $G$ and $H$ respectively, both with factor system $\sigma$, and let their characters be $\chi$ and $\psi$ respectively. Then

$$
\frac{1}{|G|} i\left(\Gamma, \Delta \uparrow_{\sigma} G\right) \sum_{g \in G_{0}} \chi(g) \chi^{*}(g)=\frac{1}{|H|} i(\Delta, \Gamma \downarrow H) \sum_{g \in H_{0}} \psi(g) \psi^{*}(g) .
$$

Proof. From equation (3.2) it follows that the left-hand-side is equal to $\left(\left|G_{0}\right| /|G|\right) i\left(D^{0}(G),\left(\Delta_{\sigma}^{\uparrow} G\right) \otimes \Gamma^{*}\right)$ and the right-hand-side is equal to $\left(\left|H_{0}\right| /|H|\right) i\left(D^{0}(H),(\Gamma \downarrow H) \otimes \Delta^{*}\right)$. But this is, from theorem 1 , equal to $\left(\left|G_{0}\right| /|G|\right) i\left(D^{0}(G),\left[(\Gamma \downarrow H) \otimes \Delta^{*}\right] \uparrow G\right)$. Finally theorem 2 completes the proof.

Theorem 4 (Transitivity of induction). Let $M$ be a subgroup of $G$ such that $H$ is a subgroup of $M$. Let $\Delta$ be a PUA representation of $H$ with factor system $\sigma$. Then

$$
\left(\Delta \uparrow_{\sigma} M\right) \uparrow_{\sigma} G=\Delta \uparrow_{\sigma} G .
$$

Proof. We have to show that $\left.\operatorname{Tr}\left\{\left(\left(\Delta_{\sigma}^{\dagger} M\right)\right)_{\sigma}^{\uparrow} G\right)(g)\right\}=\operatorname{Tr}\left\{\left(\Delta_{\sigma}^{\uparrow} G\right)(g)\right\}$ for all $g \in G_{0}$. But this is, after evaluating the traces, a straightforward calculation using the equations (2.1) and (2.2).

Let $K$ be a subgroup of $G$. Then we can factorise $G$ into double cosets: $G=$ $\Sigma_{i} H d_{i} K$. Let $K_{i}$ be defined by $K_{i}=d_{i} K d_{i}^{-1}$ and $L_{i}$ by $L_{i}=H \cap K_{i}$. If $D$ is a PUA representation of $K$ with factor system $\sigma$, then the PUA representation $D_{i}$ of $K_{i}$ with 
factor system $\sigma$ is defined by

$$
D_{i}\left(d_{i} k d_{i}^{-1}\right)=\sigma\left(d_{i} k d_{i}^{-1}, d_{i}\right) \sigma^{*}\left(d_{i}, k\right) D^{d_{i}}(k) \quad \forall k \in K
$$

Theorem 5 (Mackey's subgroup theorem). Let $D$ be a PUA representation of $K$, then

$$
D\left(\uparrow_{\sigma} G\right) \downarrow H=\sum_{i}\left\{\left(D_{i} \downarrow L_{i}\right) \uparrow_{\sigma} H\right\}
$$

Proof. Let $H=\Sigma_{j} h_{i}^{j} L_{i}$ be a left coset decomposition for $H$ with respect to $L_{i}$. Then a left coset decomposition for $G$ with respect to $K$ is given by $G=\Sigma_{i} \Sigma_{j} h_{i}^{j} d_{i} K$ (Bradley 1966). After evaluating the traces of both sides of equation (3.8) with these coset representatives the theorem is proved in a straightforward way using the equations (2.1) and (2.2).

Theorem 6. Let $\Gamma$ be a PUA representation of $H$ with factor system $\omega$ and $\Delta$ a PUA representation of $K$ with factor system $\sigma$. Then

$$
\left(\Delta \uparrow \uparrow_{\sigma} G\right) \otimes\left(\Gamma \uparrow_{\omega} G\right)=\sum_{i}\left\{\left(\left(\Delta_{i} \downarrow L_{i}\right) \otimes\left(\Gamma \downarrow L_{i}\right)\right) \uparrow_{\omega \sigma} G\right\}
$$

Proof. This theorem follows from the preceding theorems in the same way as for the case of unitary representations (Bradley 1966 and Coleman 1966).

\section{Clebsch-Gordan series of induced PUA representations}

We assume that all irreducible PUA representations of $G$ are known, and are obtained with the generalised induction procedure. So each irreducible PUA representation of $G$ with factor system $\sigma$ may be written as $\Delta^{\mu, \sigma}{ }_{\sigma} G$, where $\Delta^{\mu, \sigma}$ is an irreducible PUA representation of a subgroup $H^{\mu, \sigma}$ of $G$ with factor system $\sigma$. In this section we will consider the Clebsch-Gordan series

$$
\left(\Delta^{\mu, \sigma} \uparrow_{\sigma} G\right) \otimes\left(\Delta^{\nu, \omega} \uparrow_{\omega} G\right)=\sum_{\rho} C(\mu, \nu, \rho ; \sigma, \omega)\left(\Delta^{\rho, \omega \sigma} \uparrow_{\omega \sigma} G\right) .
$$

Here the multiplicity $C(\mu, \nu, \rho ; \sigma, \omega)$ can be written, according to equation (3.2), as

$$
C(\mu, \nu, \rho ; \sigma, \omega)=\frac{i\left(D^{0}(G),\left(\Delta^{\rho, \omega \sigma}{ }_{\omega \sigma}^{\uparrow} G\right)^{*} \otimes\left(\Delta^{\mu, \sigma}{ }_{\sigma}^{\uparrow} G\right) \otimes\left(\Delta^{\nu, \omega \uparrow}{ }_{\omega} G\right)\right)}{1 /\left|G_{0}\right| \Sigma_{g \in G_{0}}\left|\operatorname{Tr}\left\{\left(\Delta^{\rho, \sigma \omega} \uparrow_{\omega \sigma} G\right)(g)\right\}\right|^{2}} .
$$

The UA representation $\left(\Delta^{\rho, \sigma \omega} \uparrow_{\omega \sigma} G\right)^{*} \otimes\left(\Delta^{\mu, \sigma} \uparrow_{\sigma} G\right) \otimes\left(\Delta^{\nu, \omega}{ }_{\omega} G\right)$ can be decomposed by applying theorem 6 twice. Let us factorise $G$ into double cosets as follows: $G=$ $\Sigma_{i} H^{\nu, \omega} g_{i} H^{\mu, \sigma}$, let $H_{i}^{\mu, \sigma}$ be given by $H_{i}^{\mu, \sigma}=g_{i} H^{\mu, \sigma} g_{i}^{-1}$ and $K_{i}^{\mu, \nu ; \omega, \sigma}$ by $K_{i}^{\mu, \nu ; \omega, \sigma}=$ $H_{i}^{\mu, \sigma} \cap H^{\nu, \omega}$. If $\Delta_{i}^{\mu, \sigma}$ is defined according to equation (3.7) by

$$
\Delta_{i}^{\mu, \sigma}\left(g_{i} g g_{i}^{-1}\right)=\sigma\left(g_{i} g g_{i}^{-1}, g_{i}\right) \sigma^{*}\left(g_{i}, g\right) \Delta^{\mu, \sigma g_{i}}(g) \quad \forall g \in H^{\mu, \sigma}
$$

then we have

$$
\left(\Delta^{\mu, \sigma} \uparrow_{\sigma} G\right) \otimes\left(\Delta^{\nu, \omega} \uparrow_{\omega} G\right)=\sum_{i}\left\{\left(\left(\Delta_{i}^{\mu, \sigma} \downarrow K_{i}^{\mu, \nu ; \omega, \sigma}\right) \otimes\left(\Delta^{\nu, \omega} \downarrow K^{\mu, \nu ; \omega, \sigma}\right)\right) \uparrow_{\omega \sigma} G\right\} .
$$

Now suppose $G$ is factorised into double cosets for each $i$ as follows: $G=$ $\Sigma_{j} K_{i}^{\mu, \nu ; \omega, \sigma} g_{j, i} H^{\rho, \omega \sigma}$ and further $H_{j, i}^{\rho, \omega \sigma}$ and $K_{j, i}^{\mu, \nu, \rho ; \omega, \sigma}$ are defined by $H_{j, i}^{\rho, \omega \sigma}=$ $g_{j, i} H^{\rho, \omega \sigma} g_{j, i}^{-1}$ and $K_{j, i}^{\mu, \nu, \rho ; \omega, \sigma}=H_{j, i}^{\rho, \omega \sigma} \cap K_{i}^{\mu, \nu ; \omega, \sigma}$. If finally $\Delta_{i, i}^{\rho, \omega \sigma}$ is defined according to 
equation (3.7) by

$\Delta_{j, i}^{\rho, \omega \sigma}\left(g_{j, i} g g_{j, i}^{-1}\right)=(\omega \sigma)\left(g_{j, i} g g_{j, i}^{-1}, g_{j, i}\right)(\omega \sigma)^{*}\left(g_{j, i}, g\right) \Delta^{\rho, \omega \sigma g_{i, i}}(g) \quad \forall g \in H^{\rho, \omega \sigma}$

we obtain

$$
\begin{aligned}
\left(\Delta^{\rho, \omega \sigma} \uparrow_{\omega \sigma} G\right)^{*} \otimes\left(\Delta^{\mu, \sigma} \uparrow G\right) \otimes\left(\Delta_{\sigma}^{\nu, \omega} \uparrow G\right) \\
=\sum_{j} \sum_{i}\left\{\left(\left(\Delta_{j, i}^{\rho, \omega \sigma} \downarrow K\right) \otimes\left(\Delta_{i}^{\mu, \sigma} \downarrow K\right) \otimes\left(\Delta^{\nu, \omega} \downarrow K\right)\right) \uparrow G\right\}
\end{aligned}
$$

where we wrote $K_{j, i}$ instead of $K_{j, i}^{\mu, \nu, \rho ; \omega, \sigma}$ for brevity. Combining the equations (4.2) and (4.6) gives

$C(\mu, \nu, \rho ; \sigma, \omega)=\frac{\Sigma_{j} \Sigma_{i} i\left(D^{0}(G),\left\{\left(\Delta_{i, i}^{\rho, \omega \sigma} \downarrow K_{i, i}\right)^{*} \otimes\left(\Delta_{i}^{\mu, \sigma} \downarrow K_{i, i}\right) \otimes\left(\Delta^{\nu, \omega} \downarrow K_{i, i}\right)\right\} \uparrow G\right)}{1 /\left|G_{0}\right| \Sigma_{g \in G_{0}}\left|\operatorname{Tr}\left\{\left(\Delta^{\rho, \sigma \omega} \uparrow G\right)(g)\right\}\right|^{2}}$

Finally, using theorem 1 and the fact that, if $K_{j, i}^{0}=K_{j, i} \cap G_{0}$

$$
i\left(D^{0}\left(K_{j, i}\right), D\right)=i\left(D^{0}\left(K_{j, i}^{0}\right), D \downarrow K_{j, i}^{0}\right)
$$

for each UA representation $D$ of $K_{j, i}$, we obtain

$$
\begin{aligned}
& C(\mu, \nu, \rho ; \sigma, \omega) \\
&=\left.\left(\sum_{i} \sum_{i} \frac{|G|}{\left|G_{0}\right|} \frac{\left|K_{j, i}^{0}\right|}{\left|K_{j, i}\right|} i\left(D^{0}\left(K_{j, i}^{0}\right), \Delta_{j, i}^{\rho, \omega \sigma} \downarrow K_{j, i}^{0}\right)^{*} \otimes\left(\Delta_{i}^{\mu, \sigma} \downarrow K_{j, i}^{0}\right) \otimes\left(\Delta^{\nu, \omega} \downarrow K_{j, i}^{0}\right)\right)\right) \\
& \times\left(\frac{1}{\left|G_{0}\right|} \sum_{g \in G_{0}}\left|\operatorname{Tr}\left\{\left(\Delta^{\rho, \sigma \omega} \uparrow G\right)(g)\right\}\right|^{2}\right)^{-1} .
\end{aligned}
$$

\section{Selection rules}

Let us consider a physical system with symmetry group $G$ and suppose that the eigerifunctions of the Hamiltonian of the system transform under $g$ as basis functions of a PUA representation $T$ of $G$. An example of such a physical system is an electron in a crystalline solid with a uniform magnetic field; the symmetry group in this case is a Shubnikov space group (Brown 1964, Tam 1969). We are interested in matrix elements $\left(\phi_{m}^{o}, O_{k}^{\mu} \phi_{l}^{\nu}\right)$. Here the functions $\left\{\phi_{i}^{\gamma}, i=1, \ldots, \operatorname{dim} D^{\gamma}\right\}$ form a basis for the irreducible PUA representation $D^{\gamma}$ of $G$ with factor system $\sigma$ :

$$
T(g) \phi_{i}^{\gamma}=\sum_{j} D_{j i}^{\gamma}(g) \phi_{i}^{\gamma}
$$

and $O^{\mu}$ is an irreducible tensor operator transforming according to the irreducible UA representation $D^{\mu}$ of $G$ :

$$
T(g) O_{k}^{\mu} T(g)^{-1}=\sum_{l} D^{\mu}{ }_{l k}(g) O_{l}^{\mu} .
$$

Analogous to the well known selection rule for unitary representations the matrix 
element $\left(\phi_{j}^{\rho}, O_{k}^{\mu} \phi_{l}^{\nu}\right)$ is equal to zero due to the symmetry if the trivial UA representation of $G$ does not occur in the triple direct product $D^{\rho^{*}} \otimes D^{\mu} \otimes D^{\nu}$. This can be seen as follows.

If $D^{\alpha}$ and $D^{\beta}$ are two inequivalent irreducible UA representations of $G$ then we have the following orthogonality relation (Dimmock 1963):

$$
\sum_{g \in G_{0}} D_{k l}^{\alpha}(g) D_{m n}^{\beta^{*}}(g)=\sum_{g \in G_{0}} D_{k l}^{\alpha}(g) D_{m n}^{\beta^{*}}(g)=0 .
$$

This means that

$$
\sum_{g \in G_{0}} D(g)_{m n}=\sum_{g \in G_{0}} D(g)_{m n}=0
$$

for each UA representation that does not contain the trivial UA representation.

Since the operators $T(g)$ are unitary if $g \in G_{0}$ and anti-unitary if $g \notin G_{0}$ we have

$$
\begin{aligned}
\left(\phi_{m}^{\rho}, O_{k}^{\mu} \phi_{l}^{\nu}\right)= & \frac{1}{|G|}\left(\sum_{g \in G_{0}}\left(T(g) \phi_{m}^{o}, T(g) O_{k}^{\mu} T(g)^{-1} T(g) \phi_{l}^{\nu}\right)\right. \\
& \left.+\sum_{g \in G_{o}}\left(T(g) \phi_{m}^{\rho}, T(g) O_{k}^{\mu} T(g)^{-1} T(g) \phi_{l}^{\nu}\right)^{*}\right) .
\end{aligned}
$$

With the equations (5.1) and (5.2) this becomes

$$
\begin{aligned}
\left(\phi_{m}^{o}, O_{k}^{\mu} \phi_{l}^{\nu}\right)= & \frac{1}{|G|} \sum_{p, q, r}\left(\sum_{g \in G_{0}}\left[D^{\rho}(g)^{*} \otimes D^{\mu}(g) \otimes D^{\nu}(g)\right]_{p q r, m k l}\left(\phi_{p}^{\rho}, O_{q}^{\mu} \phi_{r}^{\nu}\right)\right. \\
& \left.+\sum_{g \in G_{0}}\left[D^{\rho}(g)^{*} \otimes D^{\mu}(g) \otimes D^{\nu}(g)\right]_{p q r, m k l}\left(\phi_{p}^{o}, O_{q}^{\mu} \phi_{r}^{\nu}\right)\right)
\end{aligned}
$$

and the right-hand-side is equal to zero due to equation (5.4) if $D^{\rho^{*}} \otimes D^{\mu} \otimes D^{\nu}$ does not contain the trivial UA representation of $G$. Now suppose, as in $\S 4$, that $D^{\rho}, D^{\mu}$ and $D^{\nu}$ are obtained with the generalised induction procedure: $D^{\rho}=\left(\Delta_{\sigma, \sigma}^{\rho,} G\right)$; $D^{\nu}=\left(\Delta^{\nu, \sigma}{ }_{\sigma} G\right)$ and $D^{\mu}=\Delta^{\mu} \uparrow G$.

From the results of $\S 4$ we finally obtain the result that $\left(\phi_{m}^{\rho}, O_{k}^{\mu} \phi_{l}^{\nu}\right)$ is equal to zero for all $m, k$ and $l$ if $\left(\Delta_{j, i}^{\rho, \sigma} \downarrow K_{j, i}^{0}\right) \otimes\left(\Delta_{i}^{\mu} \downarrow K_{j, i}^{0}\right) \otimes\left(\Delta^{\nu, \sigma} \downarrow K_{j, i}^{0}\right)$ (notation as in $\S 4$ ) does not contain the trivial UA representation of $K_{j, i}^{0}$ for any $i$ and $j$. Note that the order of $\Delta^{\rho, \sigma^{*}}, \Delta^{\mu}$ and $\Delta^{\nu, \sigma}$ may be chosen arbitrarily.

In a forthcoming paper we will examine this selection rule for the case where $G$ is a Shubnikov space group.

\section{Acknowledgment}

I thank Dr T Janssen for reading the manuscript.

\section{References}

Bargmann V 1954 Ann. Math., NY 59 1-46

Bradley C J 1966 J. Math. Phys. 7 1145-52

Brown E 1964 Phys. Rev. A 133 1038-44 
Coleman A J 1966 Queen's Papers in Pure and Applied Mathematics No. 4 (Kingston, Ontario: Queen's University)

Dimmock J O 1963 J. Math. Phys. 4 1307-11

Karavaev G F 1965 Sov. Phys.-Solid St. 6 2943-8

Shaw R and Lever J 1974 Commun. Math. Phys. 38 257-77

Tam W G 1969 Physica $42557-64$

Wigner E P 1939 Ann. Math., NY 40 149-204 